\title{
Modélisation mécanique des vibrations propres d'un arbre soumis aux vents, en fonction de sa morphologie
}

\author{
M Fournier 1, P Rogier 2, E Costes 3, M Jaeger 3
}

${ }^{1}$ Laboratoire de rheologie du bois de Bordeaux, UMR C123 du CNRS, BP 10, domaine de l'Hermitage, Pierroton, 33610 Cestas Gazinet;

2 Laboratoire de mécanique des solides de l'École polytechnique, 91128 Palaiseau;

${ }^{3}$ Unité de modélisation des plantes AMAP, CIRAD-GERDAT, BP 5035, 34032 Montpellier, France

(Reçu le 21 septembre 1992; accepté le 17 février 1993)

\begin{abstract}
Résumé - Dans le cadre d'un programme général sur la compréhension physique de l'interaction vent-arbre, une modélisation des premiers modes propres d'oscillation de la structure en flexiontorsion est développée. Ce modèle a pour but d'étudier les modes propres de vibration de l'arbre en fonction de sa morphologie, en utilisant les résultats d'une modélisation architecturale préliminaire. La simulation mécanique, effectuée par le code de calcul Modulet, prend en compte la flexibilité des branches. Les résultats sont d'abord analysés à propos d'un pin maritime et montrent dans ce cas l'existence de 2 modes de vibration où seul le tronc se déforme. Ce découplage entre modes du tronc et modes des branches permet d'envisager l'application d'une modélisation plus simple, fondée sur la déformation du seul tronc. L'analyse porte ensuite sur un hévéa simulé par le moteur de croissance AMAP après modélisation de l'architecture du clone. L'arbre étudié a la particularité de développer le long du tronc des axes concurrents puissants (réitérations). Les modes de vibrations calculés montrent alors des interactions complexes entre ces grosses branches et le tronc, seul le fondamental reste découplé. Les branches oscillent en même temps, engendrant des efforts importants à l'insertion, ce qui permet de comprendre la sensibilité aux vents de tels clones qui réitèrent abondamment.
\end{abstract}

mécanique de l'arbre / vent / architecture / Hevea brasiliensis / Pinus pinaster

Summary - A mechanical model of wind-induced natural sways of trees related to branching patterns. Within the scope of a more general programme about the mechanics of wind-tree interactions, a model of the first bending-twisting modes of tree sway has been developed. The model aims to relate the natural tree sways and its morphology via a preliminary architectural analysis. Mechanical simulations with the finite elements software Modulef take into account branch strains. Initial results from a Maritime pine show 2 modes where strain is observed on the trunk. As there are no significant coupling effects between the trunk modes and the branch modes a simpler model can be used based on the strain of a single trunk. Further results concern a Hevea, computed by the growth 
simulator AMAP from the architectural modelling of the clone. The morphology of the studied tree is characterized by competitive main axes growing along the trunk (reiterations). In this case, the computed modes of vibration show complicated interactions between these branches and the trunk: only the first fundamental mode remains uncoupled. Different branches sway at the same time, thus resulting in significant effort at the joint. This explains the sensitivity of such a clone (with large branches) to wind forces.

\section{tree mechanics / wind / architecture / Hevea brasiliensis / Pinus pinaster}

\section{INTRODUCTION}

Les chablis et volis posent des problèmes à l'aménagiste sylviculteur (rôle des éclaircies, choix des essences, des peuplements...), au gestionnaire ou à l'exploitant (estimation des risques, commercialisation...), ainsi que plus fondamentalement au biologiste écologue (rôle des chablis dans l'écosystème, adaptations et réactions des arbres...). Dans l'objectif d'apporter des éléments de réponse à ces questions, des études sur les phénomènes physiques mis en jeu (écoulement turbulent du vent dans le couvert, in situ et sur maquettes en soufflerie, balancement des arbres et modes de ruine) ont été développées, notamment en Allemagne (Mayer, 1987) ou en Grande-Bretagne (Cannell et Coutts, 1988). Une première approche statique de l'interaction ventarbre consiste à estimer les risques de catastrophe à partir de l'estimation de l'intensité de la poussée due à un vent stationnaire de vitesse moyenne donnée, compte tenu de la résistance de l'arbre, fonction de sa hauteur, de son diamètre et des propriétés de son bois. Cependant, les travaux récents ont montré la nécessité de considérer le caractère vibratoire de la sollicitation du vent et de la réponse de l'arbre, notamment pour comprendre l'effet des tempêtes où le problème est moins l'intensité moyenne de la poussée que son contenu énergétique dans une gamme de fréquences susceptible de déformer sensi- blement l'arbre. Certains auteurs ont entrepris l'analyse de la turbulence du vent dans le couvert (Brunet, 1985). D'autres études ont cherché à identifier expérimentalement les fonctions de transferts entre les vitesses fluctuantes des vents, les efforts supportés par les arbres et les balancements des troncs enregistrés dans divers peuplements de conifères (Wood, 1990 ; Mayer, 1987). Notamment, Mayer (1987) a montré que le balancement des troncs sous le vent s'effectue préférentiellement selon 2 basses fréquences (environ $0,15 \mathrm{~Hz}$ et $0,5 \mathrm{~Hz}$ ). Comme ces pics de réponse en fréquence ne sont visibles ni sur le spectre des vitesses incidentes, ni sur le spectre des efforts supportés, mais seulement sur le spectre des réponses (déflections ou accélérations) du tronc, ils correspondent aux modes propres d'oscillation en flexion de l'arbre. Les composantes de la poussée du vent dont les fréquences coïncident avec ces fréquences propres, caractéristiques de l'arbre (ie indépendantes de la sollicitation incidente), sont alors préférentiellement amplifiées de sorte que l'arbre se balance selon ses modes propres. Ce phénomène de résonance est susceptible de provoquer la rupture ou le désancrage, même si la vitesse moyenne du vent incident n'est pas considérable. En parallèle et en complément, nous avons choisi d'entreprendre la qualification des premiers modes propres de la structure, avec leur fréquence propre et leur déformée (Guitard, 1990). Cette étape 
permet de qualifier le comportement mécanique de l'arbre soumis à un effort dynamique. L'arbre est une structure complexe tant par sa géométrie que par son matériau, nous avons cherché à développer une modélisation de la structure et une simulation numérique des balancements propres en vue d'effectuer des tests de sensibilité et de décider des données morphologiques et rhéologiques les plus pertinentes. Une première modélisation très simple a considéré le tronc déformable comme un empilement de cylindres entrecoupé de verticilles de branches indéformables intervenant par leur masse et leur inertie de rotation (Guitard et al, 1991). Elle est ici complétée par une modélisation plus complexe tenant compte de la flexibilité des branches. Les simulations s'appuieront d'abord sur un pin maritime mesuré, puis sur un hévéa modélisé et simulé par le logiciel AMAP (atelier de modélisation de l'architecture des plantes). AMAP est un logiciel de simulation de la morphologie des plantes, fondé sur des concepts et des mesures botaniques : mise en place des entrencuds, ramification, fructification, pause, mort... décrites par des lois de probabilités calées sur des données de terrain. Il permet de décrire, dans un environnement observé, la morphologie d'un arbre réaliste d'une espèce ou d'un clone, à toutes ses étapes de croissance (nombre d'axes et position, nombre de feuilles et position...) (Jaeger, 1987 ; De Reffye et al, 1989).

\section{MATÉRIEL ET MÉTHODES}

\section{Généralités}

Les calculs ont été effectués à l'aide du code de calcul par éléments finis Modulef. Ils sont fondés sur une discrétisation de la structure et sur la méthode de Rayleigh-Ritz qui utilise la con- servation de l'énergie totale du système : sur un cycle d'oscillation, l'énergie de déformation maximale (atteinte en bout d'oscillation lorsque la vitesse de la structure - et donc son énergie cinétique - est nulle) est égale à l'énergie cinétique maximale (atteinte lorsque la structure est non déformée) ; lorsque l'on recherche les déplacements solutions sous la forme approchée de leur projection dans un espace de dimension finie (hypothèse contenue dans la méthode des éléments finis), l'égalité des 2 énergies s'écrit comme une équation matricielle :

$$
U\left(K-\omega^{2} M\right) U=0
$$

avec $U$ : vecteur déplacement ; $K$ : matrice de rigidité, faisant intervenir les raideurs (modules d'Yong, modules de torsion, inerties géométriques des sections droites) des éléments de la structures ; $M$ : matrice de masse, faisant intervenir les masses et la géométrie (inerties de rotation) des différents éléments de la structure.

Les déplacements solutions $U$ sont les solutions non nulles de cette équation, qui n'existent que pour les pulsations propres $\omega$ solutions de $\operatorname{det}\left(K-\omega^{2} M\right)=0$. Ces déplacements ne sont définis qu'à une constante multiplicative (amplitude) près.

L'amortissement n'est pas modélisé. Les travaux de Milne (1991) sur épicéa de Sitka ont montré des différences faibles entre les fréquences du mode fondamental calculées (par une méthode proche de la méthode numérique simple utilisée par D Guitard et al, 1991) et les fréquences mesurées lors d'oscillations libres (avec amortissement) du tronc préalablement fléchi.

Le code Modulef permet la prise en compte de la flexibilité des branches, du couplage entre les modes de flexion-torsion et donc le calcul d'autres modes que les 2 premiers modes du tronc approchés par la méthode numérique simple utilisee par D Guitard et al (1991). Dans l'un comme dans l'autre des 2 modèles, l'encastrement au sol est supposé parfaitement rigide. De plus, les branches flexibles sont supposées parfaitement encastrées (angle d'insertion constant au cours de la déformation) sur le tronc. La validité des résultats a d'abord été testée sur des exemples modèles (poutre droite cylindrique, conique...) en comparant les résultats obtenus avec des expressions analytiques, ce qui a notamment permis de fixer le pas de discrétisation utile (Rogier, 1991). On a choisi de limiter 
la modélisation au tronc et aux axes d'ordre 2 (branches insérées directement sur le tronc). Les ramifications d'ordre supérieur (qui interviennent notamment par leur masse) ne sont pas prises en compte.

Les données à entrer sont alors :

- la définition géométrique du tronc, considéré comme une poutre droite verticale à section circulaire, conique par morceaux ;

- la définition géométrique de chaque branche considérée comme une poutre droite cylindrique, c'est-à-dire sa hauteur d'insertion sur le tronc, son angle d'insertion (angle par rapport à la verticale), sa direction (ie son azimuth dans une projection horizontale) et son diamètre ; remarquons que le choix de branches cylindriques et non coniques est justifié par le fait de négliger les ramifications d'ordre supérieur, plutôt concentrées au bout : puisque les 2 effets (sur les masses du moins) sont du même ordre et s'opposent, il serait peu logique de décrire l'un et de négliger l'autre, alors que l'on peut espérer «rattraper" la première approximation par la seconde ;

- le module d'Young longitudinal, le module de torsion et la masse volumique des matériaux constitutifs ; les feuilles ou les aiguilles qui interviennent par leur masse sont considérées comme un surplus de masse, réparti uniformément sur chaque branche.

Le logiciel fournit alors les fréquences propres de chaque mode et la déformée associée.

\section{Simulation d'un pin maritime}

Le pin maritime déjà utilisé par Guitard et al (1991) a été calculé, dans l'objectif notamment de comparer les résultats de notre modèle plus complexe au calcul plus approché des premiers modes propres du tronc. Le pin maritime considéré est âgé de 23 ans, il comporte 21 verticilles identifiables. Pour la définition du tronc, les mesures des diamètres $D$ à chaque verticille permettent d'estimer le défilement du tronc $D(h)$ (h : hauteur), approché par 3 troncs de cônes (fig 1). Pour les verticilles de branches vivantes 10 à 16 , le nombre de branches, puis pour chaque branche, son angle d'insertion sur le tronc, son azimuth sur la circonférence, sa masse totale (bois, écorce et aiguilles), son diamètre à l'insertion et sa longueur, ont été relevés (Guitard et al, 1991 ; fig 2). La masse volumique du tronc est supposée constante, égale à $1000 \mathrm{~kg} /$ $\mathrm{m}^{3}$. Celle des branches est calculée pour chacune en tenant compte de leur masse totale. Les branches du sommet du houppier ( 3 derniers verticilles) ne sont connues que par leur masse ; elles sont donc considérées comme "collées" et quasi ponctuelles, c'est-à-dire qu'elles ont été intégrées à la masse volumique de l'élément discrétisé le plus proche de leur insertion.

Les rigidités du bois sont supposées uniformes, égales à celle du résineux de masse volumique (du bois $\mathrm{sec}$ à l'air) $500 \mathrm{~kg} / \mathrm{m}^{3}$, corrigées pour approcher celles du bois vert (Fournier, 1989), de telle sorte que $E_{L}=12450$ $\mathrm{MPa}$ et $\mathrm{G}_{\mathrm{LT}}=365 \mathrm{MPa}$.

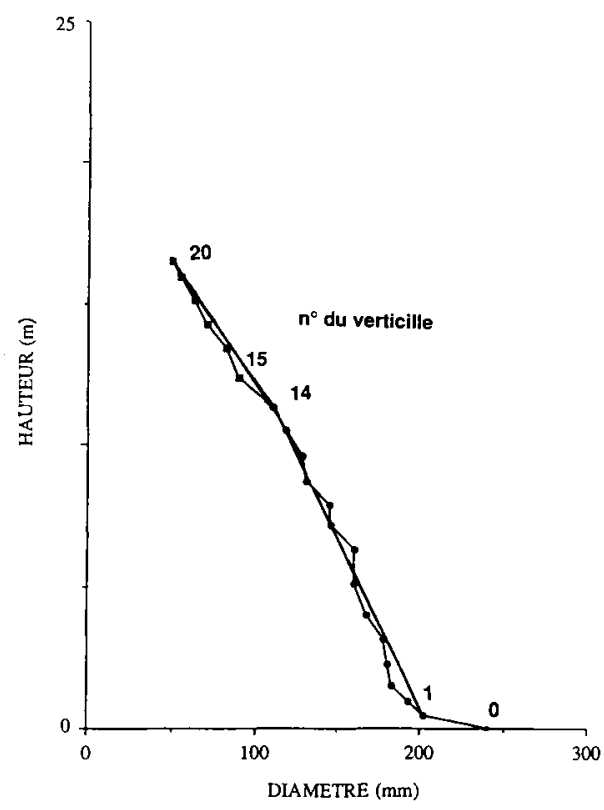

Fig 1. Description du défilement du tronc, approximé par 3 troncs de cônes : $D=0,240-$ $0,084 \mathrm{~h}$ pour $\mathrm{h}<0,455$ (empattement); $D=$ $0,200-0,008 h$ pour $0,455<h<11,320$ (verticilles 1 à $14 \mathrm{~h}$ ); $\mathrm{D}=0,217-0,010 \mathrm{~h}$ pour 11,32 $<h<17,500$ (verticilles 15 à 20 ). 


\section{Simulation d'hévéas - Utilisation des données du logiciel AMAP}

Des simulations ont été conduites sur un hévéa simulé appartenant au clone RRIM600, simulés par le logiciel AMAP. Ce clone a la particularité de développer le long du tronc des axes puissants, concurrents du tronc, qui sont des réitérations (fig 3). Leur insertion est connue pour être un point de fragilité, d'autant plus que l'axe porteur (le tronc) peut parfois être complètement dominé, et se poursuivre sous la forme d'un axe

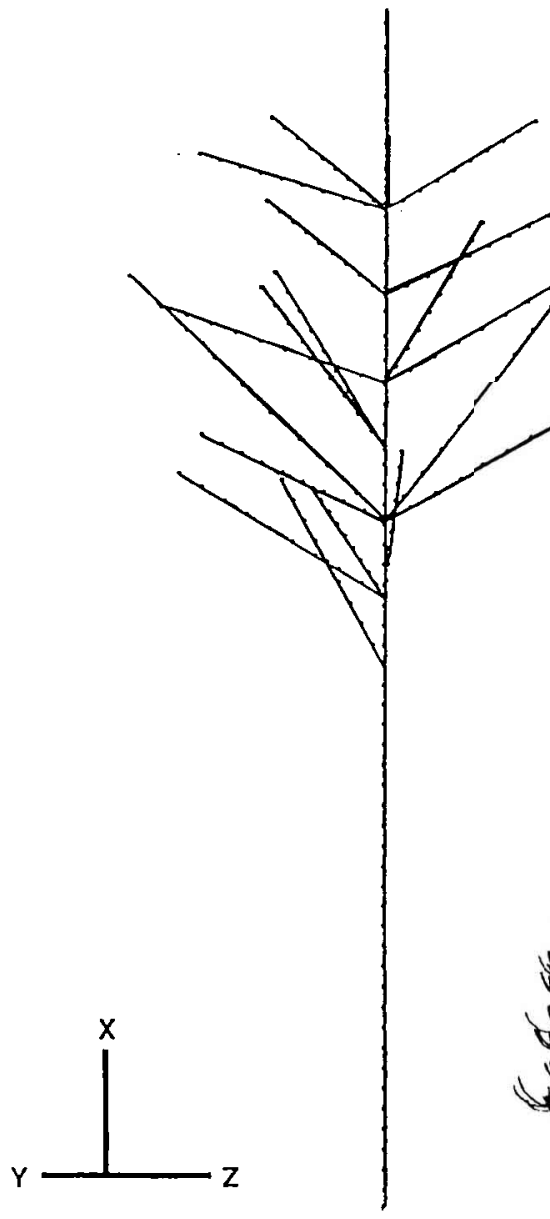

Fig 2. Morphologie et maillage du pin maritime étudié. grêle (Costes et De Reffye, 1990). Comme le montre la figure 4 (sortie du logiciel AMAP et entrée de modulef), les morphologies issues d'AMAP ont été simplifiées pour être compatibles avec la modélisation choisie, en ne conservant que l'axe porteur et les réitérations. Des concepts botaniques tels que la réitération s'avèrent donc utiles pour la modélisation mécanique : dans le cas présent, l'analyse architecturable nous permet de distinguer 2 types de branches insérées sur le tronc, et nous suggère de ne retenir que les plus significatives du point de vue de leur rigidité et de leur masse. Cette tentative de couplage entre le logiciel AMAP et l'étude mécanique de l'arbre a montré la nécessité d'inclure dans AMAP des données réalistes quant aux diamètres des axes, puisqu'actuellement on ajoute une unité constante de croissance secondaire à chaque nouvelle unité de croissance primaire. Faute de disposer de ces données, la simulation a utilisé cette hypothèse, en "calant" les valeurs absolues des diamètres sur les dimensions moyennes relatées par Polinière et Van Brandt (1965) pour le clone PR 107.
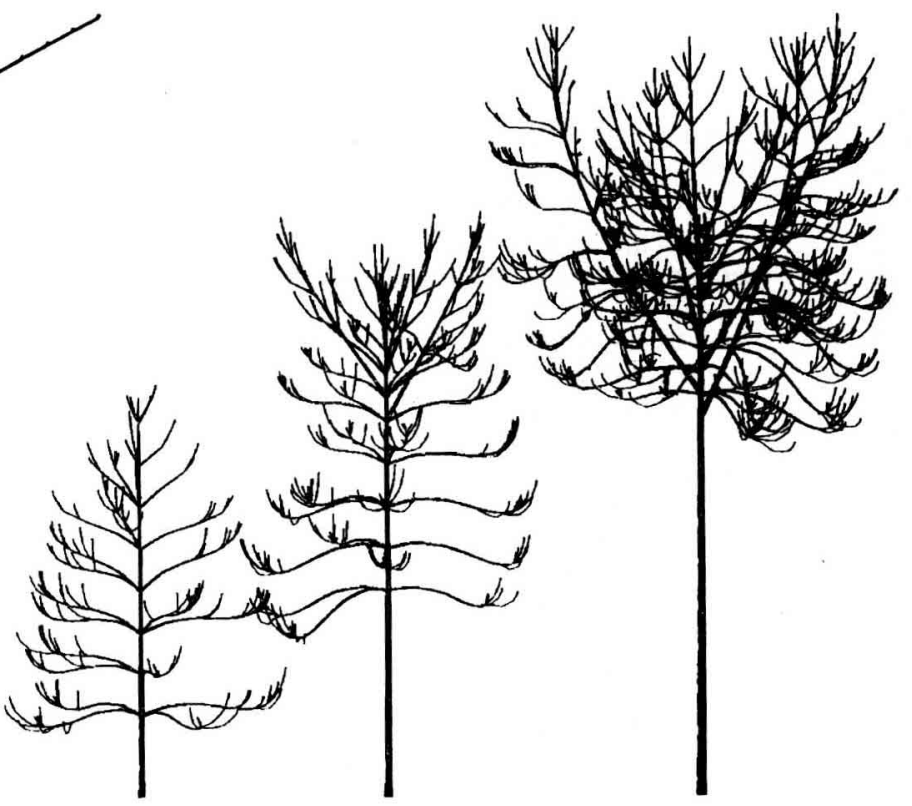

Fig 3. Simulation par le logiciel AMAP, à partir de la modélisation architecturale, de la morphologie de l'hévéa étudié à différents stades de développement. 


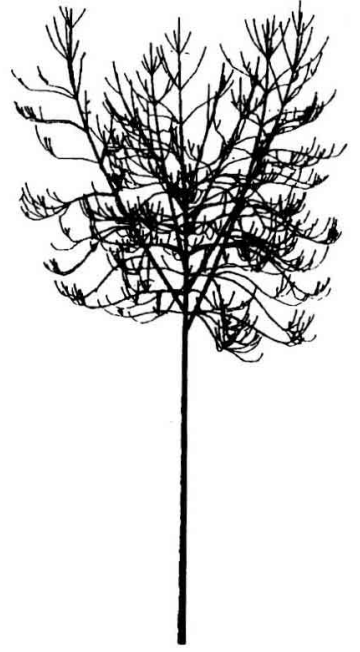

Sortie AMAP

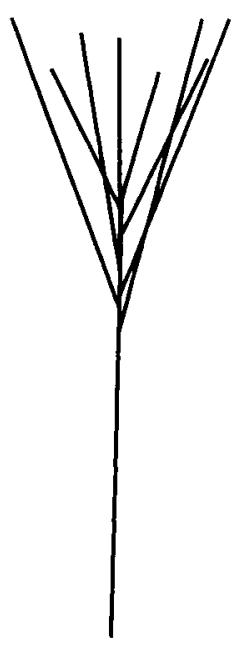

Entrée Modulef

Fig 4. Simplification de la simulation du logiciel AMAP permettant son utilisation pour le calcul mécanique.

La masse volumique du bois sec a été estimée à $650 \mathrm{~kg} / \mathrm{m}^{3}$, celle du bois vert à $1600 \mathrm{~kg} /$ $\mathrm{m}^{3}$ (humidité de $150 \%$ ).

Bien que le logiciel AMAP calcule toutes les feuilles de l'arbre, il était délicat d'utiliser ces informations en l'absence de données sur la masse de ces feuilles. La masse des feuilles a été soit négligée, soit supposée uniforme, égale à $20 \%$ de la masse des branches. On espère ainsi tester la sensibilité du modèle à ce paramètre.

Les rigidités du bois sont supposées uniformes, égales à celle du feuillu standard (Guitard, 1987), vert (Fournier, 1989), de telle sorte que $E_{L}=11800 \mathrm{MPa}$ et $G_{L T}=450 \mathrm{MPa}$.

\section{RÉSULTATS SUR LE PIN MARITIME}

Pour discuter les fréquences propres calculées par Modulef et ordonnées par ordre croissant, il est bon d'interpréter les modes en termes de déformations du tronc ou

d'une branche : ainsi, on essaiera, en observant les déformées correspondantes, de définir des fréquences $f_{i}$ et $f_{i j}$ de telle sorte que :

- $f_{0}$ corresponde au fondamental du tronc, $f_{1}$ au premier harmonique du tronc, $f_{2}$ au deuxième, etc ;

- $f_{0-01}$ corresponde à un mode de l'arbre où seulement la branche 01 vibre et est dans son fondamental... ;

Cette tentative est explicitée dans le tableau I, qui récapitule toutes les fréquences calculées par Modulef. On notera que, comme la structure ne présente pas une symétrie de révolution, chaque mode est dédoublé : par exemple, le tronc pré-

Tableau I. Premières fréquences propres du pin maritime.

\begin{tabular}{llll}
\hline Mode & Nom & \multicolumn{2}{c}{ Fréquences $(\mathrm{Hz})$} \\
& & $\begin{array}{c}\text { rer axe } \\
\text { principal }\end{array}$ & $\begin{array}{c}2^{2} \text { axe } \\
\text { principal }\end{array}$ \\
& & & \\
\hline & & & \\
00 & $f_{0}$ & 0,366 & 0,367 \\
02 & & 1,165 & 1,179 \\
04 & $f_{0-10}$ & 1,204 & 1,206 \\
06 & & 1,294 & 1,311 \\
08 & $f_{1}$ & 1,229 & 1,312 \\
10 & $f_{0-13}$ & 1,425 & 1,462 \\
12 & $f_{0-09}$ & 1,558 & 1,579 \\
14 & $f_{0-19}$ & 1,654 & 1,700 \\
16 & & 1,875 & 1,900 \\
18 & $f_{0-02}$ & 1,969 & 2,000 \\
20 & & 2,005 & 2,010 \\
22 & & 2,032 & 2,056 \\
24 & & 2,136 & 2,144 \\
26 & & 2,186 & 2,198 \\
28 & & 2,203 & 2,221 \\
30 & & 2,226 & 2,259 \\
32 & & 2,290 & 2,300 \\
34 & & 2,339 & 2,342 \\
36 & & 2,425 & 2,427 \\
38 & & 2,438 & 2,441 \\
40 & & 2,469 & 2,484 \\
42 & & 2,747 & 2,750 \\
44 & $f_{2}$ & 3,589 & 3,669 \\
& & & \\
\hline & & &
\end{tabular}


sente 2 modes fondamentaux de balancement dans 2 directions principales en flexion ; ces 2 modes correspondent à des fréquences très proches, leur composition se traduira par une trajectoire elliptique de la cime.

II apparaît immédiatement une forte imbrication entre les modes du tronc et ceux des branches. Les fréquences propres des branches seules (calculées analytiquement comme des cylindres vibrant en flexion, Rogier, 1991) sont :

$$
f_{0-i}=0,140 \frac{d}{\rho^{2}} \quad \frac{\sqrt{E}}{\rho_{i}}
$$

où $\mathrm{d}$ : diamètre, 1 : longueur, $\rho_{\mathrm{i}}$ : masse volumique de la branche i, E : module d'Young longitudinal.

On notera que l'on peut parfois retrouver ces modes individualisés des branches; par exemple pour la branche $n^{\circ} 9: d=0,0386 m ; l=2,85 m ; \rho_{09}=2319$ $\mathrm{kg} / \mathrm{m}^{3}$; et $\mathrm{f}_{0-09}$ (analytique) $=1,54 \mathrm{~Hz}$.

De fait, la figure 5 montre la déformée du mode 12, qui apparaît bien comme un mode où seule une branche vibre, le reste de la structure restant quasiment immobile.

Toutefois, les modes propres de la structure ne sont pas simplement réductibles à la somme des modes du tronc seul (les branches restant indéformables) et des modes de chaque branche parfaitement encastrée (le tronc et les autres branches restant immobiles) : des interactions complexes se manifestent lorsque les fréquences propres d'un mode du tronc seul et des modes de certaines branches seules sont proches.

Par exemple, le fondamental de la branche $\mathrm{n}^{\circ} 1 \mathrm{~d}=0,0464 \mathrm{~m}, \mathrm{l}=4,59 \mathrm{~m}$, $\rho_{03}=1284 \mathrm{~kg} / \mathrm{m}^{3}$ ) serait : $f_{0-01}$ (analytique) $=0,960 \mathrm{~Hz}$, assez proche du premier harmonique du tronc $f_{1}$.
De fait (fig 6), le mode 2 (que l'on n'ose pas appeler $f_{0-01}$ ) mêle des vibrations de la branche 1 et du premier harmonique du tronc à une fréquence intermédiaire 1,17 $\mathrm{Hz}$.

De même, les modes dont les fréquences sont comprises entre 2 et 3 sont complexes: le tronc et une ou plusieurs branches, plusieurs branches vibrent ensemble (fig 7, mode 32). Dans cet ordre d'idées, on observe, si l'on calcule les fréquences jusqu'à des valeurs plus élevées, une complexification et une imbrication des modes, qui rendent de plus en plus délicate l'interprétation.

Ces remarques étant faites, il est utile de revenir sur les modes du tronc seul (fondamental et premier harmonique), interprétés comme les modes 0 et 8 de la structure. Le fondamental, déclenché à la fréquence la plus basse (inférieure au $\mathrm{Hz}$ ), représente le balancement le plus intuitif

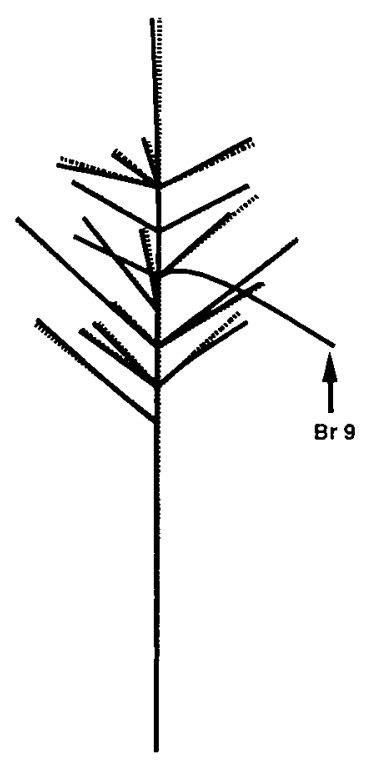

Fig 5. Déformée du mode 12 du pin maritime : la structure déformée apparaît en gras, la structure non déformée en pointillé. 


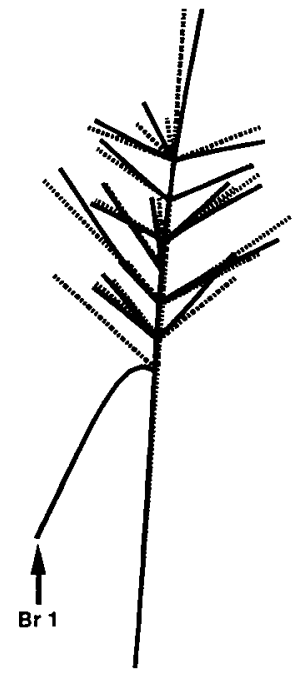

Fig 6. Déformée du mode l2 du pin maritime : la structure déformée apparaît en gras, la structure non déformée en pointillé.

(fig 8). Le premier harmonique, déclenché par une fréquence légèrement plus élevée, présente un nœud de vibration (point de déplacement nul) aux alentours des 2 tiers de la hauteur et une courbure maximale à mi-hauteur environ. Or, des observations expérimentales ont montré que ce mode peut être excité (Mayer, 1987). Le voisinage du point de courbure maximale (ventre de vibration) est alors un lieu de déformations importantes où les risques de casse existent donc. Cela pourrait expliquer pourquoi certaines peuplements (plantations de peupliers par exemple) cassent systématiquement à une certaine hauteur plutôt qu'au voisinage de l'ancrage. La sensibilité à la casse de ces individus viendrait alors moins d'une faiblesse particulière du bois à ce niveau (ou d'un défilement particulier) que d'une morpholo- gie et d'un environnement (turbulence du vent compte tenu de la densité de plantation) favorables au déclenchement de ce premier harmonique. Concernant la modélisation de ces 2 modes, la méthode approchée (branches indéformables) prévoyait $f_{0 a p}=0,395 \mathrm{~Hz}$ et $f_{1 a p}=1,51 \mathrm{~Hz}$ (Guitard et al, 1991), à comparer avec $f_{0}=0,37 \mathrm{~Hz}$ et $f_{1}=1,3 \mathrm{~Hz}\left(f_{1 \text { bis }}=1,2 \mathrm{~Hz}\right)$. On peut donc considérer que les 2 méthodes donnent des résultats comparables (à $10 \%$ près pour le fondamental, $15 \%$ pour le premier harmonique). Lorsqu'on s'intéressera aux risques de casse dans le seul tronc, la méthode approchée demeurera une excellente approximation dans le cas de faibles interactions entre les modes du tronc et ceux des branches. Un rapide calcul analytique des modes des branches devrait permettre d'apprécier les risques pris en acceptant l'approximation.

En revanche, lorsqu'on s'attachera à décrire le balancement des branches ellesmêmes (notamment le bris des fourches...), il semble utile d'utiliser une schématisation plus complexe (surtout dans l'interprétation des modes).

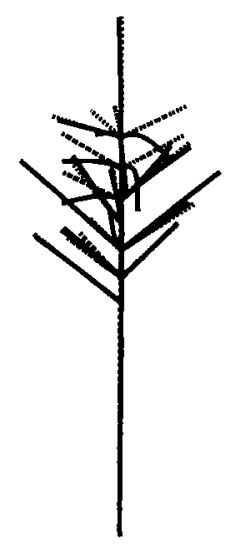

Fig 7. Déformée du mode 52 du pin maritime : la structure déformée apparaît en gras, la structure non déformée en pointillé. 


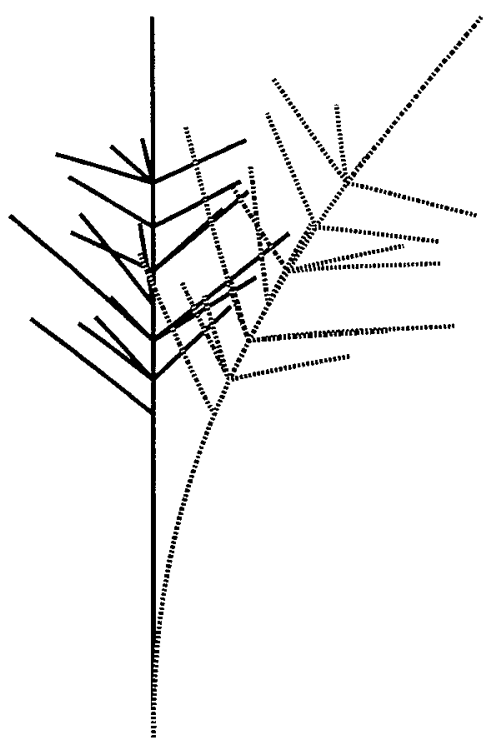

Fig 8. Déformée du fondamental (mode 0) du pin maritime : la structure déformée apparaît en gras, la structure non déformée en pointillé.

Enfin, il ne faut pas oublier que si cette démarche permet de qualifier tous les modes propres du tronc, le problème reste entier de déterminer comment l'un ou l'autre de ces modes est préférentiellement excité par un vent incident.

\section{RÉSULTATS SUR L'HÉVÉA}

Le tableau II récapitule les premières fréquences propres calculées dans le cas où les feuilles représentent $20 \%$ de la masse des branches. Dans le cas où on les néglige, l'allure des modes ne varie pas de façon perceptible, et les fréquences sont d'environ $10 \%$ supérieures.

Mis à part le fondamental (fig 10) qui correspond à un balancement du tronc seul (les branches ne se déformant quasiment pas), la simulation montre des
Tableau II. Premières fréquences propres de l'hévéa.

\begin{tabular}{|c|c|c|}
\hline \multirow[t]{2}{*}{ Mode } & \multicolumn{2}{|c|}{ Fréquences $(\mathrm{Hz})$} \\
\hline & $\begin{array}{c}f^{e r} \text { axe } \\
\text { principal }\end{array}$ & $\begin{array}{c}2^{2} \text { axe } \\
\text { principal }\end{array}$ \\
\hline 0 & 0,190 & 0,191 \\
\hline 1 & 0,411 & 0,412 \\
\hline 2 & 0,435 & 0,435 \\
\hline 3 & 0,499 & 0,499 \\
\hline 4 & 0,576 & 0,577 \\
\hline 5 & 0,686 & 0,688 \\
\hline 6 & 0,826 & 0,830 \\
\hline 7 & 1,019 & 1,026 \\
\hline 8 & 1,418 & 1,419 \\
\hline 9 & 2,544 & 2,549 \\
\hline 10 & 2,699 & 2,703 \\
\hline 11 & 2,788 & 2,840 \\
\hline 12 & 3,056 & 3,079 \\
\hline 13 & 3,517 & 3,524 \\
\hline 14 & 4,050 & 4,052 \\
\hline 15 & 4,805 & 4,829 \\
\hline
\end{tabular}

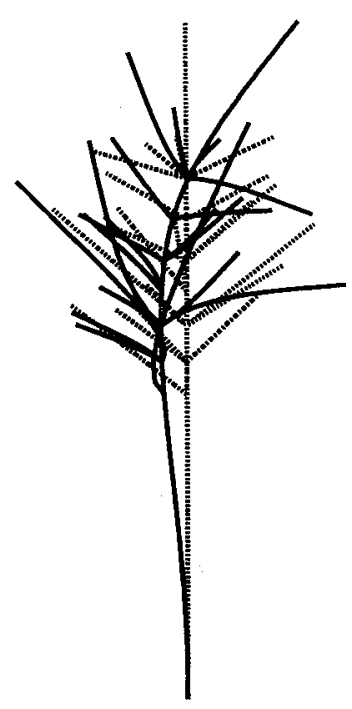

Fig 9. Déformée du premier harmonique du tronc (mode 8 ) du pin maritime : la structure déformée apparaît en gras, la structure non déformée en pointillé. 


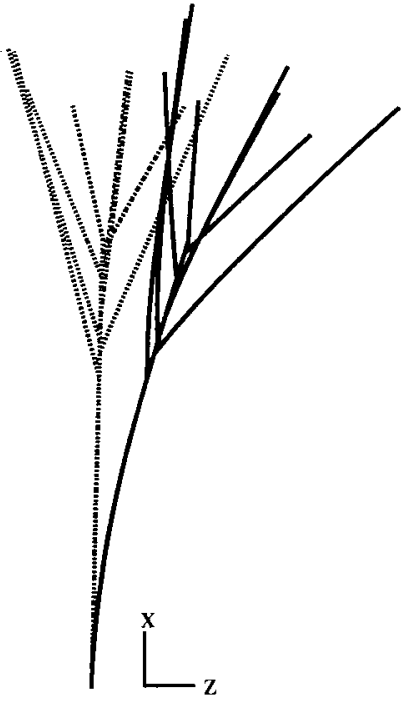

Fig 10. Déformée du mode fondamental de l'hévéa : la structure déformée apparaît en gras, la structure non déformée en pointillé.

\section{CONCLUSION}

La simulation numérique des modes propres de balancement a montré que la flexibilité des branches influe peu sur le mode fondamental de la structure qui reste une oscillation simple (un nœud à l'encastrement, un ventre au sommet) du tronc, l'amplitude de la déformation des branches restant faible par rapport à celle du tronc. Une solution approchée par des méthodes plus simples (poutre simple, branches indéformables) suffit donc à rendre compte de ce fondamental. Cela a d'ailleurs été démontré expérimentalement par Milne (1991) sur des épicéas de Sitka en plantation. En revanche, de nombreux modes de branches s'intercalent entre ce fondamental et le premier harmonique du tronc, de sorte que même si l'on se limite à des

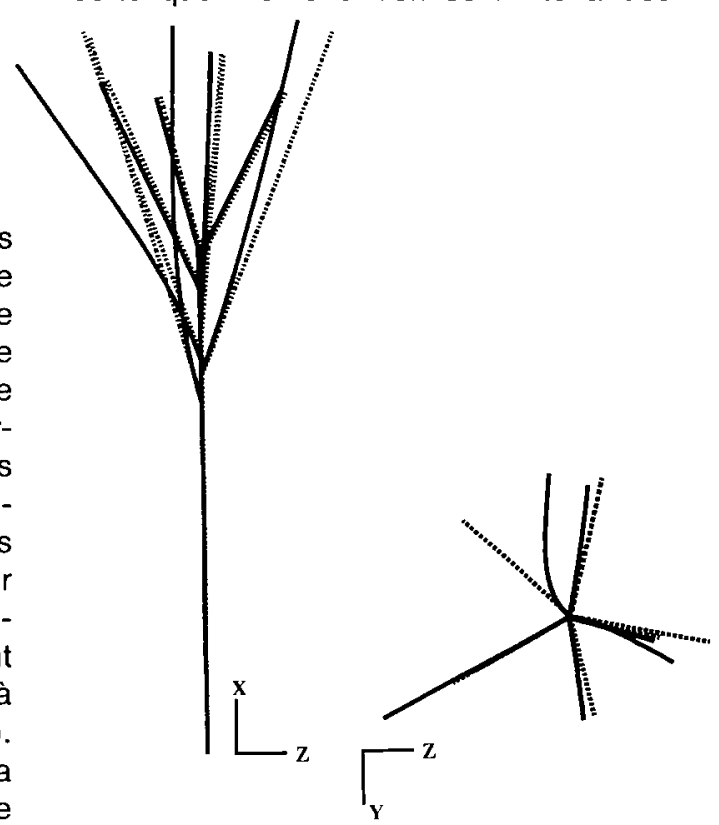

Vue de face

\section{Vue de dessus}

Fig 11. Déformée du deuxième mode de l'hévéa : la structure déformée apparaît en gras, la structure non déformée en pointillé. 


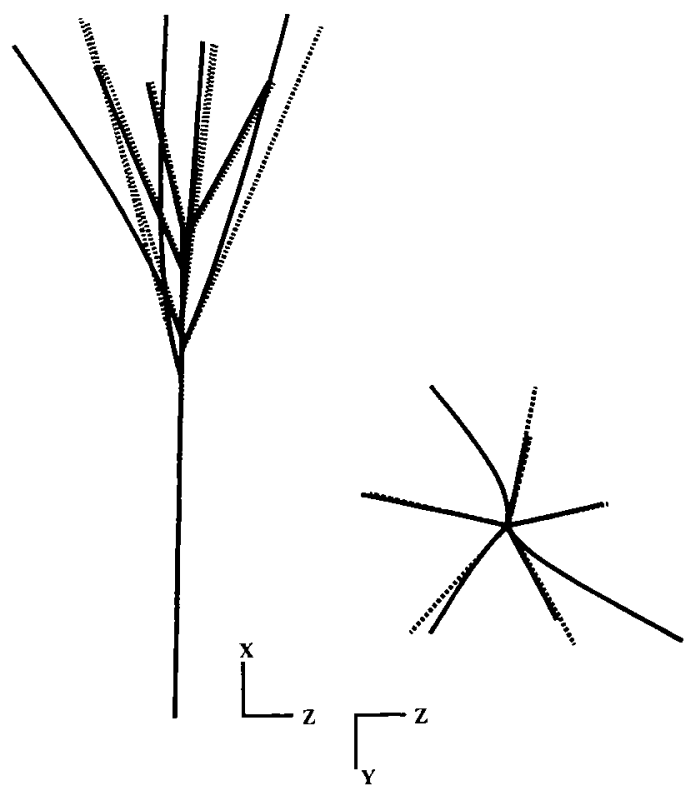

Vue de face

Vue de dessus

Fig 12. Déformée du troisième mode de l'hévéa : la structure déformée apparaît en gras, la structure non déformée en pointillé.

basses fréquences (inférieures à quelques $\mathrm{Hz}$ ), ces modes ne doivent pas systématiquement être négligés et ne peuvent être simplement calculés par découplage du tronc et du mouvement de chaque branche. Notamment, ils risquent d'être essentiels pour comprendre le balancement et la casse d'arbres fourchus fortement réitérés. II faudra donc choisir entre une méthode plus approchée focalisée sur le tronc et la modélisation plus complexe présentée ici. Notons que les 2 approches nécessitent le même type de prise de données, la méthode plus approchée, qui réclame également la connaissance de la masse et du centre de gravité des branches, n'est guère plus simple de ce point de vue.
Les simulations à partir de données issues du logiciel de simulation de la croissance des plantes ont montré l'intérêt de la modélisation architecturale pour la modélisation mécanique : par exemple, le fait de distinguer les réitérations des autres branches permet un modèle simplifié mais réaliste. Par ailleurs, elles ont souligné les points à développer : il est notamment indispensable d'inclure dans AMAP des données plus réalistes concernant la croissance secondaire des tiges de la biomasse des différents organes. Une étape ultérieure, en cours au laboratoire de rhéologie du bois de Bordeaux, consiste actuellement à la qualification expérimentale des modes, en vue d'une confrontation avec le modèle.

Enfin, il faut rappeler que le problème évoqué ici de la tenue dynamique de l'arbre aux vents, qui concerne des temps caractéristiques de l'ordre de quelques secondes, est supposé découplé en première approximation de la dynamique de croissance de l'arbre et des adaptations physiologiques. L'influence de la poussée des vents sur le volume, la morphologie des arbres et la qualité de leur bois est un tout autre problème. Toutefois, la compréhension purement physique des phénomènes hors croissance amène à remettre en cause des hypothèses simplistes telles que "le vent est assimilable à une force statique horizontale exercée à mi-hauteur " et rejoint alors, en tant qu'étude ipréliminaire, les problèmes de dynamique de la croissance (Moulia et Fournier, 1992).

\section{RÉFÉRENCES}

Brunet $Y$ (1985) Eulerian modelling of scalar transport in canopies. In: Atmospheric transport processes and plant canopies, 4-6 September 1985, Workshop CSIRO Proceedings, $21 p$

Cannel MGR, Coutts M (1988) Growing in the wind. New scientist 21, 42-46 
Costes E, De Reffye P (1990) Modélisation de l'architecture de 3 clones d'hévéa. Rapport de convention CIRAD/IRCA, $42 \mathrm{p}$

Fournier M (1989) Mécanique de l'arbre sur pied : maturation, poids propre, contraintes climatiques dans la tige standard. Thèse de doctorat de l'INPL, Nancy

Guitard D (1987) Mécanique du bois et composites. Cepadues, Toulouse, $238 \mathrm{p}$

Guitard D (1990) La réponse dynamique de l'arbre aux vents, propositions pour un programme de recherche. Deuxième séminaire: "Architecture, structure et mécanique de l'arbre" (B Thibaut, ed). LMGC, université Montpellier II

Guitard D, Castéra P, Fournier M, Radi M (1991) Comportement en flexion dynamique d'un arbre : modélisation d'un pin maritime. $3^{e}$ séminaire «Architecture, structure, mécanique de l'arbre" (B Thibaut, ed). LMGC, université Montpellier II

Jaeger M (1987) Représentation et simulation de la croissance des végétaux. Thèse de doctorat spécialité informatique, université Louis-Pasteur, Strasbourg, $156 p$
Mayer $H$ (1987) Wind-induced tree sways. Trees 1, 195-206

Milne R (1991) Dynamics of swaying of Picea sitchensis. Tree Physiol 9, 383-399

Moulia B, Fournier M (1992) Mechanics of structure and plant morphology: a critical review of plant mechanical design. Quatrième séminaire "Architecture, structure et mécanique de l'arbre" (B Thibaut, ed). LMGC, université Montpellier II

Polinière, Van Brandt (1965) Production en matière sèche et autres caractères végétatifs de greffes d'hévéa en fonction de leur âge. Rapport de recherche 1/65, Laikhe

de Reffye $P$, Edelin C, Jaeger M (1989) Modélisation de la croissance des plantes. La Recherche 20 (207), 158-168

Rogier P (1991) Réponse dynamique aux vents de la structure arbre sur pied. Rapport d'Option (C2 : sécurité et structures), promotion 1988, École polytechnique, Palaiseau

Wood JC (1990) Aeroelastic modelling of trees susceptible to windthrow damage. University of Oxford. Department of Engineering Science. Final Report, Contract CEE MA1B0063-UK(BA) 